\title{
Filigrane
}

Écoutes psychanalytiques

\section{Rencontre et discours de la méthode}

\section{Irène Krymko-Bleton}

Volume 23, numéro 2, automne 2014

URI : https://id.erudit.org/iderudit/1028926ar

DOI : https://doi.org/10.7202/1028926ar

Aller au sommaire du numéro

\section{Éditeur(s)}

Revue Santé mentale au Québec

\section{ISSN}

1192-1412 (imprimé)

1911-4656 (numérique)

Découvrir la revue

Citer cet article

Krymko-Bleton, I. (2014). Rencontre et discours de la méthode. Filigrane, 23(2), 109-124. https://doi.org/10.7202/1028926ar

\section{Résumé de l'article}

La recherche d'orientation psychanalytique ne va pas de soi à l'université, tant à cause du paradoxe de la transmission du savoir analytique par une pédagogie, qu'à cause de conceptions actuellement prédominantes sur la nature de la recherche universitaire (en tout cas, en psychologie). Le présent article fait écho à l'appel lancé il y a une dizaine d'années par Sophie de Mijolla-Mellor pour un " décentrement » de la recherche psychanalytique. L'auteure présente deux exemples de travaux universitaires où sont directement mises à profit pragmatique, analyse de discours et autres disciplines étudiant les échanges langagiers, afin de développer la recherche d'orientation psychanalytique. 


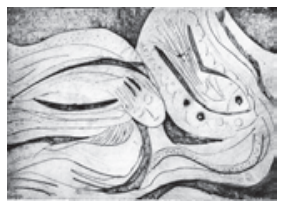

\title{
Rencontre et discours de la méthode
}

\author{
Irène Krymko-Bleton
}

La recherche d'orientation psychanalytique ne va pas de soi à l'université, tant à cause du paradoxe de la transmission du savoir analytique par une pédagogie, qu'à cause de conceptions actuellement prédominantes sur la nature de la recherche universitaire (en tout cas, en psychologie). Le présent article fait écho à l'appel lancé il y a une dizaine d'années par Sophie de Mijolla-Mellor pour un «décentrement» de la recherche psychanalytique. L'auteure présente deux exemples de travaux universitaires où sont directement mises à profit pragmatique, analyse de discours et autres disciplines étudiant les échanges langagiers, afin de développer la recherche d'orientation psychanalytique.

ly a déjà une dizaine d'années, Sophie de Mijolla-Mellor plaidait pour une approche multidisciplinaire afin de développer la recherche psychanalytique à l'université:

D’une manière générale dans le domaine de la psychanalyse, il n’y a de recherche féconde que grâce au décentrement qui va donner un nouvel axe de vision sur un objet qui a déjà été décrit ailleurs et autrement. (MijollaMellor de, 2004, 38)

Certainement désirable pour stimuler l'innovation, ce principe de décentrement ne s'en heurte pas moins immédiatement à deux obstacles: le statut institutionnel de la psychanalyse dans la recherche universitaire et le paradoxe de la transmission du savoir analytique par une pédagogie - la recherche n'impliquant pas seulement le professeur mais aussi ses étudiants.

En 1988, les professeurs psychanalystes de divers départements de l'UQÀM s'étaient regroupés dans un séminaire pour réfléchir sur leur enseignement. Informel initialement, le groupe allait devenir le GEPI (Groupe d'Études Psychanalytiques Interdisciplinaires). Ses débats m’ont laissé le souvenir de retrouvailles autour d'un désir commun de transmettre plutôt que d'enseigner. Chacun retrouvait chez les autres ce quelque chose qui paraissait différencier les membres de ce groupe de leurs autres collègues 
suscitant l'isolement chez chacun, voire, jusqu'à cette rencontre, un léger malaise. Or, au fil des ans, il m'est apparu que cette transmission était aussi possible lors de la direction de travaux de recherche.

Je propose ici des perspectives méthodologiques pour la recherche universitaire issues de la rencontre entre la psychanalyse, la sémiologie et la pragmatique linguistique; perspectives qui tiennent compte de contraintes institutionnelles en s'inscrivant néanmoins dans ce désir de transmettre.

Cet article présente donc quelques lignes directrices d'une méthodologie de recherche que je développe lors de mon travail avec mes doctorants; méthodologie qui s'appuie d'une part sur la compréhension psychanalytique du fonctionnement psychique et des situations communicationnelles et, d'autre part, sur la pragmatique linguistique pour atteindre à cette compréhension à partir du matériel verbal des entrevues.

\section{Psychanalyse, linguistique et autres sciences du langage.}

$\mathrm{La}$ rencontre psychanalytique est traditionnellement une rencontre langagière ${ }^{1}$. Or, la langue est l'objet même des études du linguiste ou du philosophe de langage; et le discours et les échanges langagiers ceux de la pragmatique linguistique, de la sémiologie, de la rhétorique, de la stylistique, de la poétique, chacune avec ses approches propres. La psychanalyse cherche à expliciter le sens de conduites humaines. La sémiologie «s'attaque aux matrices mêmes qui permettent le processus de la connaissance: le signe, le sujet, sa position socio-historique. Elle rencontre alors la psychanalyse» indique l'Encyclopaedia universalis. Toutes ces sciences pourraient donc s'avérer précieuses au développement de la recherche d'orientation psychanalytique.

Chercher à rapprocher la linguistique et la psychanalyse n'est certes pas une idée nouvelle; qu'on pense à Jacques Lacan et André Green. Néanmoins, dans ces deux cas, la collaboration n'a pas abouti. Chez Lacan, il s'agit d'un détournement et d'une transformation radicale de concepts de Ferdinand de Saussure présentés plus bas. Par ailleurs, dans sa réflexion sur le sujet (du désir inconscient), Lacan a fait usage d'une petite étude issue d'un travail colossal de deux grammairiens - Jacques Damourette et Édouard Pichon - et leur a emprunté le terme de forclusion qu'il utilisera pour décrire la structure psychotique. Certaines thèses de Lacan font penser aux propos du linguiste Émile Benveniste, comme dans la citation ci-dessous, sans que celui-ci soit nommé. En revanche, comme on va le voir, les conceptions lacaniennes sur le fonctionnement du langage ont servi de point de 
départ à l'élaboration de certaines méthodes d'analyse de discours par les auteurs qui préconisent la rencontre de la psychanalyse et de la pragmatique linguistique.

Quant à André Green, qui se pose la question du caractère performatif de la langue à propos de l'efficience de l'interprétation dans l'analyse, après avoir examiné certaines philosophies du langage et la linguistique d'Antoine Cullioli sur la question de l'interprétation (que je ne vais pas reprendre ici, puisqu'elle ne me sert pas dans le travail dont je rends compte), il dresse surtout un constat des limites de cette collaboration: ni le contenu du dire ni ses étapes pré-langagières, soubassement archaïque de la construction du sens - ce qui intéresse le psychanalyste - n'intéresse la linguistique ${ }^{2}$.

Je présenterai ici le rapprochement fait dans certains travaux de mon laboratoire entre la psychanalyse et la pragmatique linguistique, ce qui n'exclut pas la sémiologie et la rhétorique qui y trouvent aussi leur place.

\section{Pour introduire la linguistique}

Pour situer ma rencontre avec cette discipline, rencontre précédée de la lecture de travaux de Marie-Christine D’Unrug (qui date des années 1970) et poursuivie en parallèle avec le travail de Marie-Lauraine Pradelles-Monod (qui date des années 1990), je présente un bref survol de quelques étapes de théorisations linguistiques cherchant à établir la possible confluence des sciences du langage avec la psychanalyse. Ces étapes décentrent progressivement la linguistique de la seule étude de la langue, intégrant le contexte, l'effet sur l'interlocuteur et les effets de l'inconscient dans la parole. Faute de place, je procèderai à une présentation très fragmentaire et me limiterai à l'évocation des moments apparaissant les plus fertiles; j'espère que le lecteur pourra néanmoins repérer intuitivement le lien entre ces différents moments.

Sans même mentionner les philosophes stoïciens de l'antiquité grecque ou le romain Sextus Empiricus, au Xvir ${ }^{\mathrm{e}}$ siècle, Antoine Arnauld et Claude Lancelot héritent de la notion de «signe» pour penser la relation entre la langue et la logique. Puis, il faut attendre l'orée du $\mathrm{xx}^{\mathrm{e}}$ siècle pour que Ferdinand de Saussure (1857-1913) réinscrive dans une problématique plus globale, sémiologique, l'intérêt pour les mots manifesté par ces grammairiens de Port-Royal. Certes, les mots intéressent la linguistique, mais il faut les inscrire dans la langue conçue comme système de signes. Distinguant la langue de la parole, c'est-à-dire le système - la convention sociale — de son usage individuel, de Saussure peut penser la langue dans son abstraction. 
Son unité, le signe, est composite : il unit un concept et son image acoustique (retenue comme une empreinte dans le psychisme), signifié et signifiant. Un schéma illustre leur lien: le signifié, placé au-dessus du signifiant, est séparé de lui par une ligne; bien qu'ainsi séparés, signifié et signifiant ne sauraient être disjoints, comme le recto et le verso d'une feuille; c'est ensemble qu'ils constituent le signe. En revanche, le signe est disjoint de sa référence, de la chose désignée: son référent lui est extérieur.

Dans les années 1920-1940, étudiant la langue française, deux grammairiens - Jacques Damourette et Édouard Pichon (qui est aussi psychanalyste) - conçoivent une grammaire monumentale à partir de l'idée que la langue sert de fondement à la pensée. Comme cette pensée/langue n'est pas consciente d'elle-même, la tâche du grammairien consiste à amener à la conscience le système morphosyntaxique qui la caractérise.

Pour le sens commun, parler s'oppose à agir. Pour des philosophes du langage anglais, c'est plutôt en comprenant la parole comme acte que l'on éclaire son efficience. John Austin (1911-1960), en identifiant ce qui distingue les énoncés qui servent à décrire le monde (constatifs) de ceux qui réalisent une action (performatifs ${ }^{3}$ ), repère trois types d'actions opérées par le langage selon leurs effets: locutoire, illocutoire et perlocutoire ${ }^{4}$. Chaque énoncé est, à des degrés divers, affaire de relation entre interlocuteurs. Paul Grice (1913-1988), lui, s'intéresse, à la question: comment un auditeur reconnait-il l'intention d'un locuteur? Il étudie des indices permettant de saisir le sens subjectif qu'un locuteur donne à son énoncé en introduisant dans les analyses des énoncés des concepts extra-linguistiques, tels le contexte de l'échange conversationnel.

Toutes ces avancées nous rapprochent, du point de vue linguistique, de ce que va devenir l'analyse de l'échange conversationnel, c'est-à-dire, l'analyse d'une parole prononcée dans un contexte particulier d'échange ayant des effets sur l'interlocuteur qui, à son tour, tente de reconnaitre les intentions de celui qui lui parle et d'y répondre.

Dans une direction différente, Émile Benveniste (1902-1969), polyglotte et connaisseur de langues anciennes, permet le rapprochement de la linguistique et de la psychanalyse par son intérêt de linguiste pour la subjectivité. L'homme donne sens au monde à travers le langage, pense-t-il. La langue est investie d'une double signifiance, celle du signe linguistique inséré dans le système dont il fait partie et celle qui appartient au mode du discours, appropriation subjective du langage par un locuteur. Avec Benveniste s'ouvre l'étude de la parole dans une relation intersubjective mise en contexte (la 
portion de la réalité à laquelle la langue renvoie), qui doit faire objet d'un consensus entre les interlocuteurs, les transformant en co-locuteurs. La colocution implique la relation entre le je qui parle et le tu auquel il s'adresse. Une opposition seconde se créée entre ces deux premières personnes et la troisième, le il absent qui, «étant exclu[e] de la relation personnelle je-tu, a pour fonction véritable d'exprimer la non-personne» (Benveniste, 1966, 228). Une étude sur le temps dans les verbes français montre la pertinence de cette compréhension du sujet dans le discours. Benveniste différencie récit et discours, deux modalités spécifiques de l'énonciation exprimées par des marques linguistiques spécifiques. Tout discours se caractérise par «un locuteur et un auditeur, et, chez le premier, l'intention d'influencer l'autre en quelque manière» (Benveniste, 1966, 239); alors que, s'exprimant en troisième personne, la modalité du récit est impersonnelle, ne semble prise en charge par personne.

Du reste, dans cet article, l'analyse de discours selon François Flahault ${ }^{5}$ sera au fondement de l'explicitation de mon travail de recherche. Reprenant la constatation de Benveniste que parler, c'est toujours explicitement ou implicitement s'adresser à quelqu'un dans une situation particulière (un contexte situationnel), François Flahault propose une méthode d'analyse de discours cherchant à identifier «les places» que les interlocuteurs adoptent l'un par rapport à l'autre dans toute conversation, même la plus banale. Pour que la parole circule, ils doivent devenir co-locuteurs. Dans ce processus, un locuteur cherche toujours à influencer l'autre «en quelque manière».

De plus, selon Flahault, la théorie austinienne mettant en lumière les possibilités performatives de la langue est limitée à un certain type d'actes illocutoires; elle ne peut pas prendre en compte l'implicite qui soutient les échanges. Il propose donc une analyse conversationnelle mettant en lumière cet implicite et permettant l'interprétation de la relation intersubjective de locuteurs, y compris au niveau inconscient.

Le concept de "place», dont la spécificité repose sur ce trait essentiel que chacun accède à son identité à partir et à l'intérieur d'un système de places qui le dépasse, ce concept implique qu'il n'est pas de parole qui ne soit émise d'une place et convoque l'interlocuteur à une place corrélative; soit que cette parole présuppose seulement que le rapport de places est en vigueur, soit que le locuteur en attende la reconnaissance de sa place propre, ou oblige son interlocuteur à s'inscrire dans le rapport. (Flahault, $1978,58)$ (c'est moi qui souligne) 
L'acte illocutoire ajuste donc constamment la relation «qui tu es pour moi, qui je suis pour toi » : il engage l'identité du sujet qui le prononce. Chaque énonciation, chaque parole est illocutoire puisqu'elle s'appuie sur «qui je suis pour toi, qui tu es pour moi» dans le sens «tu me confirmes ou contestes dans mon dire», «tu me reconnais/tu ne me reconnais pas» (à la place que je souhaite occuper). Cette question de reconnaissance est sous-tendue par la recherche de la complétude (pour colmater le manque) du sujet.

Par ailleurs, un énoncé n'est pertinent que par rapport au contexte de son énonciation et par le cautionnement des interlocuteurs par un élément tiers, comme une situation ou un endroit où elles sont pertinentes. L'homme parlant identifie son propre discours à la réalité; un locuteur peut se trouver en porte-à-faux par rapport à ceux à qui il s'adresse, si le rapport de places dans lequel il se croit engagé (qu'il prend pour la réalité) est un rapport imaginaire. Or, la reconnaissance de la pertinence de l'énonciation du sujet (par un autre sujet ou par un groupe) est un insigne minimal de reconnaissance, de partage de cette réalité. Le niveau le plus élémentaire de la légitimation d'un discours est la reconnaissance extérieure de sa pertinence parce qu'on ne peut se reconnaitre soi-même cette pertinence. On cherche donc toujours à se faire reconnaitre par l'autre, avec qui on partage le même tissu discursif - ce qui limite son pouvoir puisqu'il est assujetti à ce même discours.

Cette reconnaissance ne peut donc arriver que si le rapport de places (le partage microsociologiquement déterminé de la langue) est tel que la parole du locuteur peut véritablement être entendue. Pourtant la connotation d'un mot peut varier d'un groupe ou d'une classe sociale à l'autre, ainsi qu'avec les expériences préalables des interlocuteurs qui déterminent les associations que les mots provoquent chez eux. Les malentendus peuvent donc être nombreux.

D'autre part, dans tout acte de parole, comme Benveniste, Flahaut différencie l'énoncé (qui peut être analysé de façon purement linguistique), de l'énonciation:

Il faut distinguer le système discursif qui sous-tend l'énoncé d'un sujet et l'énonciation (l'actualisation) de ce système, assumée par ce sujet comme étant sa parole. [...] l'existence de ce système lui étant cachée. $(1978,82)$

Toute parole est donc une constante médiation interhumaine. Flahault propose d'analyser cette parole «intermédiaire», selon quatre registres organisés hiérarchiquement (chacun englobant les précédents): 
À partir de rapport de places qui ont marqué d'une empreinte inaltérable [inconsciente] le locuteur; autrement dit, à partir de l'ensemble des paroles et relations illocutoires qui l'ont structuré inconsciemment et ont fixé le cadre de ses relations transférentielles.

Sur la base de systèmes discursifs qui correspondent à sa place dans la formation sociale à laquelle il appartient [discours idéologiques].

À partir de la place qui lui est dévolue dans le système de places qui rend possible la présente situation de production de parole et qui, inséparablement, introduit des contraintes pour le discours susceptible d'y fonctionner comme médiation entre interlocuteurs [codes, contraintes situationnelles].

Dans le cadre de la circulation et de l'affiche d'insignes déterminées par la prise des interlocuteurs dans la médiation de tel tissu discursif (médiation qui fonctionne dans la présente situation de production et d'échange de paroles), à tel moment. $(1978,138)$

Flahaut construit sa méthode d'analyse de discours idéologiques en se référant, du côté de la psychanalyse, à la conception de la parole proposée par Lacan:

Nul concept ${ }^{6}$ pourtant ne donne le sens de la parole, pas même le concept du concept, car elle n'est pas le sens du sens. Mais elle donne au sens son support dans le symbole qu'elle incarne par son acte.

C'est donc un acte, et comme tel, supposant sujet. Mais ce n'est pas assez dire que, dans cet acte, le sujet suppose un autre sujet, car bien plutôt il s'y fonde comme étant l'autre, mais dans cette unité paradoxale de l'un et de l'autre $[\ldots]$ l'un s'en remet à l'autre pour devenir identique à lui-même? $[\ldots]$

Mais si cette parole est accessible pourtant, c'est qu'aucune vraie parole n'est seulement parole du sujet, puisque c'est toujours à la fonder dans la médiation à un autre sujet qu'elle opère, et que par là, elle est ouverte à la chaine sans fin - mais non sans doute indéfini, car elle se referme - des paroles où se réalise concrètement dans la communauté humaine, la dialectique de la reconnaissance. (Lacan, 1966, 351-353) 


\section{Recherche psychanalytique et analyse de discours, rencontre formatrice}

C'est bien dans cette dialectique de reconnaissance (réciproque du chercheur et du sujet qui lui parle) que je tente d'ancrer les analyses de recherches de mes étudiants travaillant avec le matériel des entrevues, qui dès leur transcription deviennent des textes à analyser. C'est l'analyse de discours qui nous intéresse pour nos recherches et les façons qu'a la pragmatique linguistique pour l'aborder, comme chez Flahaut.

\section{Le choix de la question de recherche}

Outre la prise en compte de l'inconscient, une des principales différences entre l'approche psychanalytique et les autres approches de recherche réside dans la place centrale occupée par l'écoute (flottante) de l'analyste. Depuis Freud, interprétation et théorisation viennent toujours du travail clinique dont l'écoute est l'assise. Les questions qui se formulent, les tentatives de faire progresser la théorie ou de produire une argumentation explicative sont toujours précédées de surprises ou d'impasses dans le travail clinique ${ }^{8}$. Contrairement à toutes les autres orientations de recherche universitaire, les questions de recherche psychanalytique ne devraient pas être issues de la seule lecture d'une littérature spécialisée et ne devraient pas consister en théorisations abstraites, par des étudiants jonglant avec les notions théoriques dont ils n'ont aucune expérience personnelle. Elles devraient émerger d'une rencontre d'une écoute, faisant éclore une question: une énigme ${ }^{9}$, que la recherche tenterait d'éclairer.

Je tente donc de mettre en relief la subjectivité du chercheur dès le moment où l'étudiant-futur chercheur doit choisir sa question de recherche. Ce choix doit toujours s'ancrer à une expérience comparable à l'écoute clinique et doit faire suite à une parole porteuse d'une énigme à laquelle l'étudiant est confronté. Bien que leurs champs d'intérêt doivent être définis d'avance (et correspondre à mes propres intérêts de recherche), je demande aux étudiants de ne pas formuler leur question de recherche avant qu'elle n'émerge de l'écoute répétitive de plusieurs entrevues disponibles dans mon laboratoire, qui ont déjà fait l'objet d'analyses par d'autres étudiants. Tout en faisant des lectures préparatoires, les étudiants sont donc invités à écouter les propos recueillis tant et aussi longtemps qu'une question issue de cette écoute ne surgit pas dans leur esprit, qu'une énigme ne leur apparait pas. Aux étudiants qui ont déjà fait des stages ou qui travaillent comme psychologues, 
je demande que leur question émerge d'un dire qui les a intrigués dans les rencontres cliniques.

L'idée d'énigme est empruntée à Roland Barthes et introduite dans le laboratoire par Madeleine Sednaoui, alors étudiante au doctorat. À propos de Sarrasine d'Honoré de Balzac, Barthes pose la question de ce qu'un lecteur pourrait savoir exactement des intentions d'un auteur. Sa réponse témoigne de l'impossibilité d'une telle connaissance exacte, mais propose le processus d'analyse par lequel le texte révèle des sens cachés lors d'une lecture superficielle. La méthode présidant au travail sur le texte de Sarrasine a inspiré Sednaoui pour traquer le sens escamoté dans les entrevues.

Bien que les étudiants ne soient pas formés à l'écoute (de l'inconscient), le fait même qu'ils aient entendu quelque chose qui a échappé aux autres, et que cela ait été possible grâce à la sensibilité particulière de chacun, les introduit à la question de l'écoute (subjective) et — par la même occasion — à celle de la polyphonie de textes (dont parlait Barthes).

La question de l'écoute rejoint immanquablement celle de la demande et à partir d'elle, celle du transfert et du contre-transfert, liés — à leur tour — à l'interprétation.

\section{Demande, transfert et contre-transfert}

On considère d'habitude que si, dans la clinique, la demande est celle du sujet, dans la recherche il s'agit de la demande du chercheur. La demande du chercheur (apparemment toujours consciente) ne semble poser problème à personne: le chercheur souhaite obtenir suffisamment du matériel pour compléter sa recherche. Cette demande dominerait les entretiens. Pourtant, si au cours de l'entrevue la subjectivité du chercheur se manifeste toujours, je suis aussi d'avis que pour qu'une recherche puisse avoir lieu, la demande (consciente) du chercheur-étudiant doit rencontrer celle (ou plutôt celles) des sujets qu'il rencontre. Pour accepter de perdre son temps à répondre aux questions du chercheur, il faut bien avoir quelque chose à dire qu'on désire faire entendre! Ce quelque chose se fraie un chemin dans toute entrevue. Quelle est la nature de cette demande du sujet? Que veut-il du chercheur pour accepter de lui parler à propos de sa question de recherche? Quelle place donne-t-il au chercheur? Comment le chercheur peut-il en savoir plus sur ses propres réactions à partir de la place qu'il réclame lui-même face à celle que le sujet lui donne? Voilà des questions qui nous ramènent de proche en proche au contexte relationnel de la rencontre. Dans l'échange qui s'installe à propos de l'objet de recherche, le sujet tente d'établir un rapport 
de force avec le chercheur. De son côté, le chercheur se positionne par rapport à son interlocuteur dans l'entrevue.

L'analyse de la relation de places dans la situation de recherche remplace bien la notion de transfert dans une démarche thérapeutique. Elle éclaire tout ce qui est dit dans la rencontre. Les façons de parler qui visent à obtenir une certaine reconnaissance nous instruisent de motifs, le plus souvent inconscients, qui incitent les sujets à vouloir nous parler: on ne s'adresse pas de la même façon à un juge, à un complice, à une autorité... Le registre qui nous intéresse pour situer la relation que les sujets tentent d'établir avec le chercheur est donc le registre 4 proposé par Flahault. Les registres 2 et 3 servent de contexte à nos analyses, bien que dans plusieurs recherches, le discours idéologique soit tellement prévalent et serve d'une telle défense massive, que son analyse en tant que mécanisme de défense trouve toute sa pertinence.

Bien entendu, l'étudiant-chercheur tente aussi d'établir un équilibre de places lors de l'entrevue qu'il mène, effort couronné de plus au moins de succès selon les entrevues et plus au moins heureux quant aux visées de la recherche. Cet effort, qu'on pourrait partiellement assimiler au contre-transfert, est tout autant analysable que celui du sujet qui lui accorde l'entrevue. Le prendre en compte peut donc être utile dans le processus interprétatif.

\section{L'entrevue: texte à analyser}

Une fois transcrite, l'entrevue devient texte. Les possibilités qui s'ouvrent dans un travail sur ce texte compensent, par la minutie qu'ils permettent, le caractère fragmentaire du matériel dont dispose le chercheur. Ce travail consiste en allées et venues entre différents niveaux d'analyse et la littérature pertinente sur le sujet.

Pour rendre le matériel plus abondant, ce texte peut être complété par des écrits dans un cahier laissé à la disposition du sujet où il peut noter, après l'entrevue, les idées ou les rêves qui lui sont venus a posteriori et qu'il souhaite faire parvenir au chercheur. Pour cerner quelque chose de la vérité du sujet d'où émane la demande de reconnaissance - donc quelque chose de l'inconscient —, bien que l'analyse des places offre toujours quelques pistes de réflexion, plusieurs autres procédés doivent intervenir. Nous nous appuyons donc sur l'analyse minutieuse des façons de dire, sur l'argumentation, sur l'organisation du récit et sur les glissements dans le fil du récit - l'acte illocutoire ne se confondant pas avec ces aspects du discours qui l'accompagnent. Toutefois, malgré des étapes obligées et la référence 
commune aux analyses de discours, chaque recherche, selon la question posée et le matériel recueilli, doit trouver sa propre méthode pour dégager le sens du dire.

Dans notre matière verbale, il s'agit de relever, dans un premier temps et comme il est d'usage, les thèmes abordés dans l'entrevue (explicites, mais aussi implicites). En revanche, par opposition à bien des recherches, même qualitatives, ces thèmes ne sont pas traités comme des faits rapportés (la soidisant réalité objective), mais toujours comme tentatives d'une élaboration subjective en lien avec le thème annoncé de la recherche et dans le contexte de maintien d'un équilibre de places avec le chercheur. Si la valeur informative directe des entrevues est d'habitude assez pauvre, l'étude des articulations dans le texte, de logiques d'enchainement thématique, de l'évolution des façons de dire, se révèle rapidement riche de sens.

En effet, la formulation d'une parole dans un contexte d'échange permet une certaine élaboration de la pensée. Cette élaboration procède par les chaines associatives retraçables et situables non seulement dans le contexte global de l'entrevue, mais aussi dans les contextes plus particuliers de leur apparition à l'intérieur du texte. Elles révèlent l'importance des préoccupations des sujets et dévoilent ce qui échappe à leur effort de contrôle conscient sur leur dire. De plus, les figures rhétoriques (utilisées spontanément pour obtenir tel ou tel effet sur l'auditeur), les procédés stylistiques qui peuvent être complexes ou se limiter à l'usage d'expressions toutes faites, permettent entre autres de juger de la distance que le sujet tente d'établir entre lui-même et son interlocuteur ou entre le thème qu'il aborde et lui-même.

Nous avons puisé notamment dans le travail de la linguiste et psychanalyste Marie-Christine D’Unrug qui a établi dans les années 1970 (un peu avant la publication de la méthode de Flahault, et traduisant un intérêt similaire pour l'utilisation de la parole singulière dans le champs social) des correspondances entre certaines façons de dire et les mécanismes inconscients de défense apparaissant dans le discours. Après avoir passé en revue toutes les façons d'analyser des entrevues d'enquête non-directives ou semidirectives pratiquées à l'époque dans le champ des sciences sociales, elle défend le point de vue qui fait prévaloir l'analyse de l'énonciation sur celle de l'énoncé. Pour D'Unrug tout acte discursif est un processus d'élaboration de la pensée. Comme la parole adressée à un interlocuteur se doit d'être logique, toute contradiction interne (conflit, incertitude, etc.) va se traduire dans le discours par des tentatives de sa neutralisation. Ce sont les figures de style et les procédés narratifs qui reflètent ces tentatives. Lorsqu'elles 
sont réussies, le discours garde ou retrouve sa cohérence et sa structure. Lorsqu'elles échouent, le discours se désorganise, on y trouve des illogismes, des répétitions, etc.

Les figures [de style] déterminent les méandres du discours $[\ldots]$ alternances, plus au moins marquées, entre des moments d'équilibre et des moments de déséquilibre, voire de conflit spécifique, indicatifs de l'efficacité des «actions» entreprises par le locuteur.

On voit combien forme et contenu sont liés, à un niveau, au-delà de la phrase, où l'on admet que les déterminations strictement linguistiques ne jouent plus. [...] [Les détours du locuteur] à l'intérieur du code linguistique représentent des efforts pour rester cohérent. (D’Unrug, 1974, 230)

Si une psychanalyse permet que, par la médiation de la parole, des résidus infantiles soient intégrés dans la personnalité adulte, c'est aussi par la parole

[...] que peut se faire la médiation entre une problématique vécue [qui peut être liée aux contraintes sociales et idéologiques autant qu'aux traumatismes anciens] et le sujet social chez lequel elle s'actualise. (D'Unrug, 1974, 232)

Les différences de formulation apparaissant entre différents thèmes, dans l'évolution du même thème ou entre différents moments de l'entrevue, permettent donc de suivre la dynamique du discours. L'écart entre la nature d'un énoncé et les modalités singulières de son énonciation révèle son importance pour le locuteur, la charge émotive qu'il peut recéler, indique l'impression que le locuteur tente de susciter chez son interlocuteur ou encore un glissement de sens qui révèle un processus sous-jacent dont le locuteur n'est pas tout à fait conscient ou se défend.

En effet, le sujet n'est jamais le maitre parfaitement conscient de son dire. L'inconscient se glisse immanquablement dans les interstices de toutes ces formulations langagières ${ }^{10}$.

Comme le rappelle Marie-Lorraine Pradelles-Monod ${ }^{11}$ :

L'intérêt que Freud accorde aux «manières de dire» forme la substance de ses premières publications. Que ce soit des récits de rêves, des lapsus, des oublis, des mots d'esprit, ils lui offrent le matériel linguistique, la «matière 
verbale» par lesquels l'inconscient se manifeste [...] (Pradelles-Monod, 1999, 79)

S’appuyant sur l'axe temporel dégagé par Benveniste, elle fonde sa démarche interprétative sur l'idée que l'inconscient se repère dans toute activité langagière et que les marques énonciatives peuvent être repérées dans le texte selon l'axe temporel. Des entrevues entre trois générations de femmes sont soumises à des analyses minutieuses par étapes. Ces étapes ne sont pas prévues d'avance et leur nécessité se présente au fur et à mesure de l'analyse.

Y sont isolés des fragments, décontextualisés pour fin d'analyse, mis ensuite en rapport avec des séquences plus longues et avec le texte dans son ensemble.

Les modes d'approche de ces unités sont variés: repérage des termes employés et de leur occurrences, repérage des tournures grammaticales utilisées et de leur fréquence d'emploi, repérage des relations syntaxiques entre les éléments (ordre des mots, modalités verbales, mode d'articulation d'un ou plusieurs fragments entre eux), repérage des co-occurrences d'éléments dans une même séquence [...] (Pradelles-Monod, 1999, 93)

L'investigation elle-même s'appuie sur la notion rhétorique d'écart:

Est recherché:

— l'écart repérable par rapport à des données factuelles [...]

- l'écart introduit par des variations entre des fragments apparemment identiques sur le plan du contenu sémantique [...]

— l'écart produit par la polysémie de la langue: équivoques, ambiguïtés de sens $[\ldots]$

Ce va et vient permet la mise à jour des éléments sur lesquels s'appuie l'interprétation. (Pradelles-Monod, 1999, 94)

Un énoncé est dans cet axe un «évènement évanouissant» qui ne se reproduit pas deux fois à l'identique. Le glissement dans l'acte d'énonciation, ce «déjà énoncé » qui se répète et évolue dans le texte, permet de découvrir ce que le «sujet énonciateur» communique au chercheur. 


\section{Conclusion}

Il existe une opposition frontale entre la recherche se référant à la psychanalyse et les méthodes de recherche enseignées dans un département de psychologie. Chaque question de recherche requiert un choix approprié de procédés pour recueillir les éléments nécessaires à la formulation d'une interprétation. Chaque recherche requiert donc l'élaboration de sa propre méthode. Tous mes étudiants ne suivent pas toutes les étapes du schéma méthodologique présenté ici. Néanmoins, leur travail vise à éclairer sur tout ce qui témoigne de la division du sujet, de la conflictualité de son univers intrapsychique et inter-relationnel, jusqu'au niveau inconscient qui le détermine. Les interprétations qui en résultent sont toujours hypothétiques, mais en est-il autrement dans le processus thérapeutique? Ces caractéristiques sous-tendent le statut institutionnel singulier, voire malcommode de la psychanalyse dans la recherche universitaire.

De plus, fonctionner dans un tel système demande beaucoup de souplesse de la part des étudiants, souvent d'ailleurs confus tant que leur propre recherche n'arrive pas à les propulser vers une démarche, qui s'impose d'elle-même à eux. Prendre conscience qu'une façon de procéder standardisée n'existe pas et qu'il leur faut trouver eux-mêmes les façons de faire les mieux adaptées à la question qu'ils se posent et au matériel dont ils disposent déstabilise les étudiants. La professeure que je suis ne peut que leur indiquer une multitude de références parmi lesquelles il leur faudra non seulement choisir, mais à partir desquelles il leur faudra souvent innover.

Ils développent leurs analyses pas à pas, chaque étape appelant le choix de la suivante. Cela s'oppose radicalement à ce qu'on leur demande dans le cadre de l'examen doctoral, qui porte justement sur le choix de méthode préalable à «l'expérimentation». Il serait sans aucun doute préférable que l'approche psychanalytique à la recherche soit reconnue (et pas seulement tolérée) et enseignée de façon à la fois cohérente et diversifiée. Comme ce n'est toutefois pas encore le cas, le tribut d'angoisse que paient mes étudiants au paradoxe de la transmission du savoir analytique à travers une pédagogie n'a-t-il pas une double valence: à la fois dommageable mais aussi, et dans le même temps, une initiation universitaire à l'incertitude psychanalytique?

\section{Notes}

1. Je traite ailleurs du travail avec des dessins d'enfants.

2. Les choses ont évolué, à preuve: Marie-Anne Paveau (2006) Les prédiscours. Sens, mémoire, cognition, Paris: Presses de la Sorbonne nouvelle. 
3. Ainsi «Je te baptise, au nom [...]» constitue l'acte de baptême, sous réserve que le reste non-langagier du rituel soit respecté; alors que «je t'ai baptisé» ou «il te baptise» ne sont que des constatifs.

4. De ces trois caractères du dire, le premier indique simplement l'acte de dire quelque chose; le deuxième indique que l'énoncé place linguistiquement les interlocuteurs l'un par rapport à l'autre; et le troisième indique l'effet psychologique du dire sur l'auditeur.

5. Philosophe et anthropologue, directeur de recherche au Centre de recherches sur les arts et le langage au CNRS.

6. Il s'agit ici de la critique du concept saussurien.

7. Ce n'est pas très loin de thèses de Benveniste non exposées ici, pour qui la relation linguistique Je-Tu instaure la possibilité d'émergence de la subjectivité.

8. Je mets ici à part le travail de Freud — et de ceux qui l'ont suivi — à partir de textes (comme celui du président Schreber) ou des œuvres littéraires. Une telle pratique ouvre largement la porte à bien des applications de la psychanalyse. Ce qui est pertinent pour mon travail personnel dans ce genre de pratique est la place accordée à l'écriture. Pour moi, cette place constitue une sorte de "point de capiton» entre la psychanalyse et les autres disciplines qui travaillent sur la langue.

9. Pour cette question de l'énigme, nous renvoyons le lecteur à $S / Z$ de Roland Barthes ou aux thèses qui s'en sont inspirées, lesquelles figurent dans les références.

10. Les limites de l'espace alloué pour les articles ne permettent pas de présenter de façon plus systématique et approfondie les méthodes d'analyse utilisées.

11. À l'époque de sa thèse, professeure au Laboratoire de psychopathologie de l'Université Louis Pasteur à Strasbourg.

\section{Références}

Arrivé, M., 2005, Langage et psychanalyse, linguistique et inconscient: Freud, Saussure, Pichon, Lacan, Limoges, Lambert-Lucas.

Austin, J., 1962, How to do Things with Words, Oxford, Oxford University Press (traduction française: 1970 Quand dire, c'est faire, Paris, Seuil).

Benveniste, E., 1966-74, Problèmes de linguistique générale, T. I, Paris, Gallimard.

Damourette, J., Pichon, É., 1911-1940, Des mots à la pensée. Essai de grammaire de la langue française, Paris: Ed. d'Arthrey, 1968-1971

Flahault, F., 1978, La parole intermédiaire, Paris, Seuil.

Green A., 1997, Le langage au sein de la théorie générale de la représentation, in PinolDouriez, M., Pulsion, Représentations, Langage, Lausanne, Delachaux et Niestlé.

Kristeva, J., 2012 Émile Benveniste, un linguiste qui ne dit ni ne cache, mais signifie. Préface à Émile Benveniste, Dernières Leçons, Collège de France (1968-69), Paris, Gallimard.

Lacan, J., 1966, Fonction et champ de la parole et du langage en psychanalyse, Écrits, Paris, Seuil. Mijolla-Mellor de, S., 2004, La recherche en psychanalyse à l'Université, Recherches en psychanalyse, 1, (internet: DOI: 10.3917/rep.001.0027)

Mosès, S., 2001/4, Émile Benveniste et la linguistique du dialogue, Revue de métaphysique et de morale, $\mathrm{n}^{\circ}$ 32, Paris, Presses universitaires de France, 509-525.

Pradelles-Monod, M-L., 1999, La construction du lien de filiation entre trois générations de femmes: Repères pour une analyse clinique d'entretiens de recherche, thèse présentée pour l'obtention du doctorat d'État, Strasbourg.

Sednaoui, M., 1996, L'alliance paternelle dans le processus du travail d'accueil au cours de la grossesse terminée par un accouchement prématuré pour causes idiopathiques. Thèse de doctorat, UQAM. 
Turner M.-L., 2001, Conflits maternels entourant le sexe du bébé à naitre et fantasmes mortiferes concernant ce bébé: une double menace pour des femmes fragilisées par un risque d'accouchement prématuré. Thèse de doctorat, UQAM.

Turner M-L., Krymko-Bleton I., 2002, Risque d'accouchement prématuré et fantasmes mortifères: la maternité et les contes. Dialogue, recherches cliniques et sociologiques sur le couple et la famille, $n^{\circ} 156$, Érès, Paris.

Unrug Marie-Christine d', 1974, Analyse de contenu et acte de parole. De l'énoncé à l'énonciation. Paris, Éditions universitaires. 